\title{
Zytopathologische Ergussdiagnostik in der berufsgenossenschaftlichen Begutachtung von Asbest-assoziierten malignen Mesotheliomen und Lungenkarzinomen
}

\author{
Pleural Effusion Cytology of Asbestos-Associated Malignant Mesothelioma and Lung Carcinoma in the Diagnosis \\ of Occupational Diseases by the Statutory Accident Insurance Funds
}

Autoren

Institute
S. Biesterfeld ${ }^{1}$, M. Schramm ${ }^{1}$, A. Schad ${ }^{2}$, N. Pomjanski ${ }^{1}$

${ }^{1}$ Schwerpunkt Cytopathologie (Leiter: Prof. Dr. med. S. Biesterfeld), Heinrich Heine-Universität Düsseldorf

${ }^{2}$ Institut für Pathologie (Leiter: Prof. C. J. Kirkpatrick, M.D., Ph.D., D.Sc.), Johannes Gutenberg-Universität Mainz eingereicht 28.10 .2013

akzeptiert 30.1 .2014

Bibliografie

DOI http://dx.doi.org/

10.1055/s-0034-1365157

Online-Publikation: 10.3.2014

Pneumologie 2014; 68: 270-276

(c) Georg Thieme Verlag KG

Stuttgart · New York

ISSN 0934-8387

Korrespondenzadresse

Prof. Dr. med.

Stefan Biesterfeld

Schwerpunkt Cytopathologie

Universitätsklinikum Düsseldorf

Moorenstr. 5

40225 Düsseldorf

Stefan.Biesterfeld@med.uni-

duesseldorf.de

\section{Zusammenfassung}

Bei Lungenkarzinomen und malignen Mesotheliomen ist die Bildung von Pleuraergüssen oft der erste Hinweis auf eine Tumorerkrankung. Da die Ergüsse relativ früh im diagnostischen Ablauf punktiert werden, steht Material zur zytopathologischen Tumordiagnostik häufig bereits zur Verfügung, bevor histologische Probenentnahmen erfolgt sind. In der hier präsentierten Studie wird über Erfahrungen mit der zytopathologischen Ergussdiagnostik in der berufsgenossenschaftlichen (BG) Begutachtung von sieben Patienten berichtet, bei denen keine histologischen Gewebeproben aus Lungen- oder Pleurabiopsien vorlagen. Untersucht wurden im Mittel acht Sedimentausstriche von auswärts vorbefundeten Pleuraergüssen bei sieben Männern (mittleres Alter: 81,7 Jahre) mit jeweils langjähriger Asbestexposition und in einigen Fällen BG-anerkannter Asbestose. Nach der üblichen konventionellen zytopathologischen Untersuchung wurden fallbezogen die Methoden der Immunzytochemie, der DNA-Bildzytometrie, der AgNOR-Analyse sowie der Fluoreszenz in situ-Hybridisierung angewandt.

Sechs der sieben Fälle ließen sich gutachterlich abschließend lösen. In drei Fällen lag ein malignes Mesotheliom im Vollbeweis vor, in zwei Fällen eine Pleurakarzinose eines Lungenkarzinoms. Bei einem mit Mesotheliom-Verdacht punktierten Patienten wurden Zellen eines hochmalignen Non-Hodgkin-Lymphoms (NHL) nachgewiesen und ein Mesotheliom ausgeschlossen. Nur ein Fall blieb diagnostisch offen; hier wurde später auswärts nach dem Tod des Patienten ein malignes Mesotheliom durch Autopsie gesichert. In allen sieben Fällen ist die jeweilige BG unserer Beurteilung gefolgt und hat die fünf Fälle von Mesotheliomen und Lungenkarzinomen als Berufskrankheiten anerkannt sowie den NHL-Fall abgelehnt. Der diagnostisch ungeklärte Fall wurde zunächst nicht anerkannt, sondern erst nach dem

\section{Abstract \\ $\nabla$}

Pleural effusion often represents the first clinical symptom of lung carcinoma and malignant mesothelioma. As pleural punctation is performed quite early in the diagnostic procedure, effusion cytology frequently gives the first evidence about the presence of tumour cells and tumor histogenesis. In this study, we report on seven cases which were evaluated in our institution for the Employers' Liability Insurance Association, based solely on cytology findings.

The mean age of the seven patients with a given long-term asbestos exposure during their working life was 81.7 years. On average eight smears per patient were investigated. In addition to routine cytology, immunocytochemistry, DNA image cytometry, AgNOR-analysis and fluorescence in situ hybridization were applied in a case-specific way. The results were interpreted against the clinical and occupational history of the respective patient.

Definitive diagnosis could be made in six cases. In three of them, the diagnosis of malignant mesothelioma was made. Two cases were diagnosed as malignant effusion due to metastatic lung cancer. In one case, cells of high-grade Non-Hodgkin's lymphoma (NHL) were diagnosed and a malignant mesothelioma was excluded. In the last case, malignant mesothelioma could not be diagnosed unequivocally by cytology. In all seven cases, our interpretation was accepted by Employers' Liability Insurance Association. The five mesothelioma or lung cancer cases were accepted as asbestosassociated occupational disease, while the NHL case was rejected. In the last case, malignant mesothelioma was diagnosed later by autopsy, and the case was retroactively accepted as occupational disease.

Cytology-based tumor diagnosis including adjuvant methods is a useful and reliable approach in cases of asbestos-associated tumours. Acceptance 
Vorliegen des Autopsieergebnisses rückwirkend als Berufskrankheit akzeptiert.

Die Ergebnisse zeigen, dass bei Anwendung entsprechender Methoden eine zytopathologische Erkennung und Typisierung von Tumorzellen in Pleuraergüssen nicht nur im allgemeinen möglich ist, sondern dass auch die Anerkennung einer Asbest-assoziierten Tumorerkrankung als Berufskrankheit im Rahmen einer BG-Begutachtung auf diese Weise erbracht werden kann. Entsprechend könnte bei diesen oft sehr alten und multimorbiden Patienten bei aussagekräftigem Pleuraerguss im Grunde auf eine histologische Diagnosesicherung ganz verzichtet werden.

\section{Einleitung}

\section{$\nabla$}

Auch wenn die Verwendung von Asbest als Werkstoff in Deutschland seit nunmehr gut 20 Jahren (1993) verboten ist, werden aufgrund der im Durchschnitt 25-40 Jahre [1-3] dauernden Latenzzeit zwischen Asbestkontakt und Tumorentstehung noch mehrere Jahrzehnte bedeutende Zahlen von Neuerkrankungen von Asbest-bedingten malignen Mesotheliomen und Lungenkarzinomen zu beobachten sein [4]. Zahlen für das Jahr 2007 zeigen, dass dabei in Deutschland Asbest-assoziierte Lungenkarzinome $(n=828)$ nicht wesentlich seltener auftreten als Asbest-assoziierte maligne Mesotheliome $(n=948)$ [5]. Bezieht man diese Zahlen auf die Gesamtfälle von ca. 46000 neu diagnostizierten Lungenkarzinomen im Jahr 2006 [6] und auf die derzeit auf ca. 1000 Fälle pro Jahr geschätzte Zahl von malignen Mesotheliomen, so ist insbesondere beim malignen Mesotheliom ein Asbestbezug in der großen Mehrzahl der Fälle epidemiologisch evident. In der Berufskrankheitenverordnung [7] sind entsprechend in Anlage 1 neben der Asbestose (BK-Nr.4103) auch die Asbest-assoziierten Lungenkarzinome (BK-Nr.4104 bzw. 4114) bzw. malignen Mesotheliome (BK-Nr.4105) aufgenommen.

Viele Fälle von Lungenkarzinomen und malignen Mesotheliomen sind mit der Bildung von Pleuraergüssen vergesellschaftet, nicht selten als Leitsymptom und erstem Hinweis auf eine solche Erkrankung. Da die oft voluminösen Ergüsse in aller Regel zur Entlastung des Pleuraraumes und zur Verbesserung der respiratorischen Situation der Patienten früh punktiert werden, steht zytologisches Material zur Tumordiagnostik in vielen Fällen bereits in Form von Ergusspunktaten zur Verfügung, bevor histologische Probenentnahmen erfolgt sind. Hieraus leitet sich die Frage ab, ob man eine Histologie in den Fällen, in denen der Erguss Tumorzellen enthält oder zumindest vermuten lässt, erzwingen muss oder ob man nicht mit der zytologischen Diagnostik, ggf. ergänzt um adjuvante Methoden, diagnostisch auskommt.

In der hier vorliegenden Arbeit berichten wir über gutachterliche Erfahrungen für verschiedene Berufsgenossenschaften (BG) aus den Jahren 2010-2013. Allen sieben Fällen ist dabei gleich, dass keine Histologie vorlag und sich somit die gesamte Beurteilung rein auf zytologische Präparate gründete, die primär in anderen Instituten für Pathologie vorbefundet worden waren. Fünf Fälle bezogen sich auf die Fragestellung nach einem malignen Mesotheliom, zwei auf die Unterscheidung zwischen einer Pleurakarzinose eines Lungenkarzinoms und der eines anderweitigen metastasierten Karzinoms. Insgesamt lässt sich feststellen, dass sich sechs der sieben Fälle abschließend lösen ließen und die gutachterlichen Voten von den Berufsgenossenschaften als hinreichend für eine versorgungsrelevante Entscheidung über das Vorliegen einer Berufskrankheit akzeptiert wurden. of occupational disease on the basis of cytological diagnoses even by the Employers' Liability Insurance Association helps avoid invasive pleural or lung biopsies in cases with an unequivocally positive effusion cytology of lung cancer or malignant mesothelioma.

\section{Material und Methoden}

Bei den Patienten handelte es sich um sieben Männer im Alter zwischen 68 und 92 Jahren (Mittelwert: 81,7 Jahre) mit malignen bzw. auf Malignität verdächtigen Pleuraergüssen. Zwei von ihnen waren zum Zeitpunkt der Begutachtung bereits verstorben. Zur Verfügung standen Angaben zur Anamnese sowie die relevanten Arztberichte und Befundtexte, in zwei Fällen ergänzt um CDs mit Ergebnissen der radiologischen Bildgebung. Bei allen Patienten war eine längere berufliche Asbestexposition dokumentiert, wobei konkrete Zahlenangaben zur jeweiligen Asbestfaserbelastung nicht vorlagen, möglicherweise der Tatsache geschuldet, dass jeweils nur Auszüge der BG-Akte, aber nicht der gesamte Aktenfaszikel, von der jeweiligen $B G$ vorgelegt worden waren.

Ferner wurden 3-14 zytologische Präparate (Mittelwert: 8) aus der durchgeführten Zytodiagnostik der Pleuraergüsse von den beteiligten Instituten für Pathologie zur Verfügung gestellt. Neben May-Grünwald-Giemsa-(MGG), Papanicolaou-(Pap), PASbzw. mit Hämatoxilin und Eosin (HE) gefärbten Präparaten wurden in einigen Fällen auch immunzytochemische Färbungen mit übersandt.

\section{Konventionelle Zytologie}

Die zytologischen Präparate wurden lichtmikroskopisch durchgemustert und nach den üblichen Kriterien der Ergusszytologie interpretiert [8-10]. Hierbei ist häufig insbesondere die Abgrenzung zwischen neoplastischen und reaktiven mesothelialen Zellen schwierig, weil es hierfür keine harten, sondern lediglich relative Kriterien mit recht breiter Überschneidung der morphologischen Merkmale gibt. Hierzu gehören insbesondere die bei malignen Mesotheliomen oft höhere Zelldichte, die Neigung zum Wachstum in dreidimensionalen großen, oft sogenannt maulbeerartig oder Morula-artig aufgebauten Zellverbänden, eine häufig höhere Kerngrößenvariabilität, manchmal auch verbunden mit Atypien und Bildung von Makronukleolen, sowie das mögliche Auftreten vielkerniger mesothelialer Riesenzellen. Zellen von Adenokarzinomen lassen sich andererseits recht gut aufgrund von Zellatypien und der typischen Lagerung in drüsenartigen Verbänden identifizieren.

\section{Ergänzende Untersuchungsmethoden}

Die ergänzende Diagnostik stützte sich auf in unserer Einrichtung erprobte adjuvante Verfahren [11-12], die im Kontext bereits vorhandener Anwendungen der Primärbefunder und dem sonstigen aktuellen Stand der Diagnostik abgestuft und ggf. nacheinander eingesetzt wurden.

Immunzytochemie. Zur Differenzierung zwischen mesothelialen Zellen und epithelialen Zellen wurden insbesondere eine Calretinin- und eine berEP4-Immunzytochemie angewandt. Ein Immunphänotyp [berEP4-/Calretinin+] wurde als indikativ für 
Tab. 1 Klinische Kenndaten und diagnostisches Vorgehen bei der BG-Begutachtung von sieben Fällen maligner Pleuraergüsse. MGG=May-Grünwald-GiemsaFärbung, Pap = Papanicolaou-Färbung, PAS= PAS-Färbung, HE= HE-Färbung. Zu den übrigen Methoden vgl. Text. In den Fällen mit der Fragestellung eines malignen Mesothelioms (Fälle 1 - 5) wurde die Sicherheit dieser Diagnose nach der Klassifikation des Europäischen Mesotheliompanels (vgl. [17]) bei der Abschlussdiagnose in eckigen Klammern angegeben.

\begin{tabular}{|c|c|c|c|c|c|c|}
\hline Fall & $\begin{array}{l}\text { Alter des } \\
\text { Patienten } \\
\text { (Jahre) }\end{array}$ & Ausstriche & $\begin{array}{l}\text { Relevante } \\
\text { auswärtige } \\
\text { Färbungen }\end{array}$ & $\begin{array}{l}\text { Relevante auswärtige } \\
\text { Immunzytochemie }\end{array}$ & Adjuvante Methoden & Abschlussdiagnose \\
\hline 1 & 92 & 10 & MGG, Pap, PAS & Calretinin, berEP4 & $\begin{array}{l}\text { Immunzytochemie: WT1, TTF-1, Napsin A } \\
\text { DNA-Bildzytometrie, AgNOR-Analyse, } \\
\text { 9p21-FisH }\end{array}$ & Mesotheliom [A] \\
\hline 2 & 69 & 10 & MGG & $\begin{array}{l}\text { Calretinin, } \\
\text { Thrombomodulin }\end{array}$ & $\begin{array}{l}\text { Immunzytochemie: berEP4 } \\
\text { DNA-Bildzytometrie, AgNOR-Analyse, } \\
\text { 9p21-FisH }\end{array}$ & Mesotheliom [A] \\
\hline 3 & 87 & 14 & MGG, Pap, PAS & $\begin{array}{l}\text { Calretinin, } \\
\text { Thrombomodulin }\end{array}$ & $\begin{array}{l}\text { Immunzytochemie: berEP4 } \\
\text { [Weitere adjuvante Methoden waren aus } \\
\text { technischen Gründen nicht anwendbar.] }\end{array}$ & Mesotheliom [A] \\
\hline 4 & 90 & 8 & MGG, Pap & Calretinin, CEA & $\begin{array}{l}\text { Immunzytochemie: Calretinin, WT1, } \\
\text { CD68, berEP4 } \\
\text { DNA-Bildzytometrie, AgNOR-Analyse, } \\
\text { 9p21-FisH }\end{array}$ & $\begin{array}{l}\text { Mesotheliom nicht } \\
\text { sicher [C] }\end{array}$ \\
\hline 5 & 89 & 3 & MGG, Pap & - & $\begin{array}{l}\text { Immunzytochemie: CD3, CD20, Myelo- } \\
\text { peroxidase, Calretinin, berEP4, CD68 } \\
\text { Klonalitätsanalysen für B- und T-Zellen }\end{array}$ & $\begin{array}{l}\text { wenige Zellen eines } \\
\text { hochmalignen B-NHL; } \\
\text { kein Mesotheliom [E] }\end{array}$ \\
\hline 6 & 68 & 3 & MGG & berEP4 & Immunzytochemie: TTF-1, Napsin A & Lungenkarzinom \\
\hline 7 & 77 & 8 & HE, PAS & - & Immunzytochemie: Calretinin, berEP4, TTF-1 & Lungenkarzinom \\
\hline
\end{tabular}

mesotheliale Zellen interpretiert, derjenige von [berEP4+/Calretinin-] als Nachweis einer epithelialen Genese und damit einer Pleurakarzinose. In diesen Fällen wurden danach eine TTF-1bzw. auch eine Napsin A-Immunzytochemie eingesetzt, um das Vorliegen eines primären Lungenkarzinoms zu klären. Alle bei den Falldarstellungen erwähnten immunzytochemischen Anwendungen aus der auswärtigen Primärbefundung wurden im Text mit einem Symbol $\left(^{*}\right)$ markiert.

DNA-Bildzytometrie. Die DNA-zytometrische Untersuchung auf DNA-Aneuploidie diente in den Fällen, in denen es um ein malignes Mesotheliom ging, dem Nachweis eines typischen DNA-Verteilungsmusters für maligne Mesotheliome und somit der Differenzierung zwischen einer malignen und einer reaktiven Läsion. Die Untersuchungen wurden mit einem TV-Bildanalysesystem MotiCyte DNA (Fa. Motic, Xianmen/Wetzlar) an 300 auffälligen Zellen durchgeführt und nach den Vorgaben des Consensus-Reports der European Society for Analytical and Cellular Pathology (ESACP) [13] interpretiert. DNA-Aneuploidie wurde entsprechend bei Nachweis einer DNA-Stammlinie außerhalb des Bereiches von $2 c+/-0,2 c$ bzw. außerhalb von $4 c+/-0,4 c$ oder beim Auftreten von DNA-Einzelwerten oberhalb von $9 \mathrm{c}$ angenommen.

AgNOR-Analyse. Die Durchführung einer $\mathrm{AgNO}_{3}$-basierten Versilberungsreaktion zur Darstellung der Nukleolus-organisierenden Regionen (NORs) wurde an mesothelialen Läsionen gemäß den Empfehlungen der European Society of Pathology (ESP) [14] eingesetzt und diente ebenfalls der Unterscheidung zwischen einem malignen Mesotheliom und reaktiven mesothelialen Veränderungen. An den nach der Versilberungsreaktion erkennbaren schwarzen nukleolären AgNOR-Strukturen von 100 auffälligen Zellen wurden die Cluster und Satelliten [15] separat gezählt. Fanden sich als Mittelwert $\geq 2,5$ Satelliten und $\geq 4,5$ Signale aus Clustern und Satelliten, wurde das Präparat als AgNOR-positiv interpretiert [12].

Fluoreszenz-in-situ-Hybridisierung (FisH). Eine Untersuchung auf das Vorliegen einer Deletion an der Region 9p21, in welcher unter anderem auch das p16-Gen liegt, wurde an den mesothelia- len Läsionen mithilfe einer FisH-Untersuchung mit einer regionären (9p21) und einer Zentromersonde (CEP 9) (Abbott Vysis, Downers Grove, USA) nach Vorgaben des Herstellers durchgeführt. Pro Fall wurden 100 auffällige Zellen fluoreszenzmikroskopisch ausgewertet. Als Schwelle für Positivität galt der Nachweis von mindestens fünf Zellen mit homozygoter Deletion bzw. von mindestens 15 Zellen mit heterozygoter Deletion [12,16].

\section{Ergebnisse}

Im Folgenden sind die wesentlichen anamnestischen, klinischbildgebenden und morphologischen Gesichtspunkte ( $\bullet$ Tab.1) zu den sieben Patienten wiedergegeben, wobei manchmal aus methodischen Gründen bzw. inhaltlichen Gründen (Fall 5) von der oben skizzierten Vorgehensweise etwas abgewichen wurde. In unseren Beurteilungen für die Berufsgenossenschaften wurde in den Fällen 1-3 sowie 6 und 7 die Anerkennung einer Berufskrankheit empfohlen und von den BGs auch akzeptiert. In den Fällen 4 und 5 erwiesen sich die Kriterien hierfür als nicht erfüllt.

\section{Fragestellung malignes Mesotheliom}

Fall 1: Ein 92-jähriger, zum Gutachtenzeitpunkt noch lebender ehemaliger Betriebsingenieur mit langjährigen Tätigkeiten unter Asbestexposition, seit 33 Jahren außer Dienst. Im 9-monatigen Verlauf nachlaufende einseitige Pleuraergüsse mit zunehmender Wandverbreiterung der betroffenen Pleurahöhle; ansonsten in der Bildgebung (Thorax-CT, Abdomen-CT, Sonografie) keine Hinweise auf Raumforderungen. Im initialen Pleuraerguss auswärts „Verdacht auf malignes Mesotheliom“, im zweiten Erguss neun Monate später (an anderer Stelle unter Unkenntnis der Voruntersuchung) „malignes Mesotheliom“.

Zytologisch bei der Begutachtung typisch maulbeerartige Verbände und atypische Einzelzellen, aber keine Hyaluronsäureniederschläge. Immunzytochemisch kräftige Calretinin*- und WT1-Positivität bei minimaler berEP4*-Positivität und TTF-1- 
bzw. Napsin A-Negativität. DNA-bildzytometrisch Nachweis einer aneuploiden Stammlinie bei 3,0c. Atypische AgNOR-Verteilung. In der FisH-Untersuchung kein Nachweis einer 9p21-Deletion.

In Würdigung aller Befunde lag somit ein malignes Mesotheliom im Vollbeweis vor (Mesotheliom A nach der Klassifikation des Europäischen Mesotheliompanels, vgl. [17]).

Fall 2: Ein 69-jähriger, noch lebender Mann mit 38 Jahre umfassender beruflicher Asbestexposition im Baugewerbe, seit 16 Jahren pensioniert. Im Abdomen-CT-Zufallsbefund eines basalen Pleuraergusses links. Auswärts zytologische Diagnose eines epitheloiden Mesothelioms, das bildgebend als Stadium cT1c eingestuft wurde; unter Polychemotherapie mit zunächst drei Zyklen Cisplatin/Alimta stabiler Befund.

Bei der Begutachtung von prätherapeutisch gewonnenen Ausstrichen neben Blut und Entzündungszellen maulbeerartig aufgebaute Verbände sowie kolonnenartig angeordnete Mesothelzellgruppen, die Zellen mit unterschiedlich großen, teils deutlich hyperchromatischen und entrundeten Zellkernen. Ausgeprägte Hyaluronsäurepräzipitate. Immunzytochemisch schwache bis mäßige Calretinin*-Positivität sowie Thrombomodulin*-Positivität bei berEP4-Negativität. DNA-bildzytometrisch Nachweis zweier aneuploider Stammlinien bei 2,5c und 4,5c. Atypische AgNOR-Verteilung. Mittels FisH Nachweis einer heterozygoten Deletion an der Region 9p21 in sechs atypischen Zellen.

Unter Zugrundelegung aller Befunde lag auch hier ein malignes Mesotheliom im Sinne eines Vollbeweises vor (Mesotheliom A).

Fall 3: Ein 87-jähriger, bei Begutachtung noch lebender Mann mit ca. 20 Jahre dauernder, im Aktenauszug der BG als „massiv“ bzw. „extrem“ bezeichneter beruflicher Asbestexposition als Maschinist in Kraftwerken bis vor 38 Jahren; seit 29 Jahren pensioniert. Vor ca. einem Jahr Nachweis eines kleinen einseitigen Pleuraergusses, zytologisch auswärts „Tumorzellen eines epitheloiden malignen Mesothelioms“; ferner Tumorzell-freies Bronchialsekret.

Bei der Begutachtung im Pleuraerguss sehr kompakte, Morula-artige Verbände aus atypischen, epitheloid wirkenden Zellen mit vergrößerten, hyperchromatischen Kernen mit prominenten Nukleolen und wenig Zytoplasma. Typische Hyaluronsäurepräzipitate. Immunzytochemisch Calretinin*-Positivität und berEP4-Negativität als Ausdruck einer mesothelialen Differenzierung. Aufgrund der sehr kompakten Lagerung der Zellen war die Anwendung von DNA-Bildzytometrie, AgNOR-Analyse und FisH weder an diesem, noch an einem neuen, von uns bei der behandelnden Klinik erbetenen Ergusspunktat technisch möglich.

Zusammenfassend wurde im Kontext aller vorliegenden Befunde ein malignes Mesotheliom diagnostiziert, da reaktive pleurale Veränderungen bereits morphologisch nicht mehr in Betracht kamen und andere Malignome bei dem ermittelten Immunprofil ausschieden (Mesotheliom A).

Fall 4: Ein 90-jähriger, bei Begutachtung noch lebender Patient mit über 37 Jahre zurückliegenden, langjährigen Asbest-exponierten Tätigkeiten als Ingenieurassistent auf See, die seitens der BG in die Kategorie einer "gefährdenden Tätigkeit“ nach §2 VbgBK eingeordnet wurde. Seit ca. einem Jahr einseitiger Pleuraerguss, zytologisch bei Erstpunktion auswärts mit Veränderungen, die „an eine Mesothelzellproliferation, differenzialdiagnostisch auch an ein malignes Pleuramesotheliom" denken ließen, bei Zweitpunktion mit „sicherer Malignität“, wobei aufgrund einer hohen Hyaluronsäurekonzentration in der Ergussflüssigkeit [18] vom Vorliegen eines Mesothelioms ausgegangen wurde.
Zytologisch bei der Begutachtung vor einem leicht entzündlichen Hintergrund auffällige Zellelemente, die in dichten Verbänden bzw. in einzelzelliger bis kolonnenartiger Anordnung lagen und leicht größenvariable Kerne aufwiesen. Im Hintergrund Hyaluronsäurepräzipitate. Immunzytochemisch bei Calretinin*- und WT1-Positivität sowie CEA*- und berEP4-Negativität Identifikation der auffälligen Zellen als mesothelialer Genese. DNA-bildzytometrisch normales DNA-Verteilungsmuster mit einer Stammlinie bei 2,05c und kleinem Verdopplungsgipfel bei 4c. Unauffällige AgNOR-Verteilung. In der FisH-Untersuchung keine 9p21Deletion.

Zusammenfassend ließ sich somit kein eindeutiger Nachweis eines malignen Mesothelioms erbringen (Mesotheliom C), wobei diese Interpretation im Widerspruch blieb zu der in Richtung eines Mesothelioms weisenden massiven Erhöhung des Hyaluronsäurewertes im Erguss. Eine Anerkennung als Berufserkrankung erfolgte daraufhin seitens der BG zum damaligen Sachstand nicht. Nach dem Tod des Patienten wurde dann auswärts durch eine Obduktion ein malignes Mesotheliom aber noch im Vollbeweis gesichert.

Fall 5: Ein 89-jähriger, bereits verstorbener Mann mit langjährigen Tätigkeiten unter Asbestexposition im Kessel- und Kraftwerksbau, seit 29 Jahren pensioniert. Zwei Jahre vor Pleurapunktion erster CT-Verdacht auf malignes Mesotheliom bei Pleuritis calcarea. In der Beurteilung des Pleuraergusses auswärts „Nachweis auffälliger blastenartiger, am ehesten lymphoider Zellen, aber kein Hinweis auf ein malignes Mesotheliom“. Im Thorax-CT jetzt einseitige Kompressionsatelektase bei multiplen kalkhaltigen Pleuraplaques beidseits und diaphragmal betonter ovalärscholliger Raumforderung, „differentialdiagnostisch in erster Linie fortgeschrittenes malignes Mesotheliom“.

Zytologisch bei der Begutachtung zellreiche Ausstriche mit einigen Granulozyten, kleinen unauffälligen Lymphozyten, regelrechten, einzeln liegenden Mesothelzellen und einigen auffälligen, vom morphologischen Bild in erster Linie als lymphatischer Genese anzusehenden Zellelementen; insgesamt kein Mesotheliomäquivalent. Immunzytochemisch waren die auffälligen Zellen positiv für den B-Zell-Marker CD20, aber negativ für den T-Zell-Marker CD3 sowie auch für Myeloperoxidase, Calretinin, berEP4 und CD68. In der daraufhin auswärts veranlassten molekularpathologischen Untersuchung ergab sich ein Nachweis einer monoklonalen B-Zell-Population und einer polyklonalen T-Zell-Population.

Insgesamt waren die Befunde somit indikativ für das Vorliegen weniger Zellen eines hochmalignen Non-Hodgkin-Lymphoms der B-Zell-Reihe; Hinweise auf ein malignes Mesotheliom ergaben sich bei der Begutachtung nicht (Mesotheliom E).

\section{Fragestellung Lungenkarzinom}

Fall 6: Ein 68-jähriger, bereits verstorbener multimorbider Patient mit seit 10 Jahren BG-anerkannter Asbestose im Sinne „Asbest-bedingter Rippenfellveränderungen“, bislang ohne festgestellten Rentenanspruch; keine Angaben zur Berufsanamnese. Neun Monate vor dem Tod radiologisch hochgradiger Verdacht auf ein Lungenkarzinom des rechten Mittellappens bei Rundherd; ferner Verdacht auf Hirnmetastasen. Im Pleuraerguss auswärts Nachweis von Tumorzellen eines Plattenepithelkarzinoms, „vereinbar mit einem Primärtumor der Lunge“. Keine weitere Abklärung aufgrund des schlechten Allgemeinzustandes und der fehlenden klinisch-therapeutischen Konsequenz.

Bei der Begutachtung fanden sich vor einem entzündlichen Hintergrund wenige Zellen eines nicht-kleinzelligen Karzinoms, bei 
denen man bei uns ein adenosquamöses Karzinom favorisiert hätte. Am einzigen für immunzytochemische Untersuchungen technisch geeigneten Ausstrich TTF-1-Negativität und minimale Napsin A-Positivität der Tumorzellen.

Insgesamt ließ sich somit ein nicht-kleinzelliges Karzinom bestätigen; nachdem bei dem Patienten im klinischen Verlauf neben dem Lungenrundherd auch mittels Bildgebung keine anderen für einen Primärtumor in Frage kommenden Raumforderungen erhoben worden waren und der Tumortyp gut mit einem Lungenkarzinom vereinbar war, ergab sich aus gutachterlicher Sicht unter Berücksichtigung aller Gesichtspunkte am Vorliegen eines primären Lungenkarzinoms kein vernünftiger Zweifel.

Fall 7: Ein 77-jähriger, noch lebender Mann mit seit acht Jahren BG-anerkannter Asbestose (10\% MdE). Keine Angaben zu beruflicher Tätigkeit und Dauer der Asbestexposition. Seit einem Jahr bestehende Ergussbildung links sowie eine $1,5 \mathrm{~cm}$ messende Raumforderung im linken Unterlappen. Bildgebend gesicherter Nachweis von Pleuraplaques. Zytologisch wurden neben einer unauffälligen pleuralen Biopsie mit dem Bild einer chronisch-fibrosierenden Entzündung frei beiliegende atypische Zellgruppen gesehen, die $\mathrm{CK}^{*}$-, berEP4- und CEA*-positiv sowie TTF- ${ }^{*}$ - und Calretinin*-negativ waren und somit als Adenokarzinom interpretiert wurden.

Bei der Begutachtung sah man von Blut umschlossene atypische, drüsig imponierende Zellgruppen mit mäßiger Kerngrößenvariabilität und fokaler Vakuolisierung des Zytoplasmas. Das beschriebene Immunprofil kontrastierte dabei deutlich zu der berEP4Negativität und Calretinin*-Positivität des mit erfassten Mesothels der Pleura, sodass sich auch bei uns die Diagnose eines Adenokarzinoms ergab. Weiterführende Untersuchungen wurden nicht durchgeführt; der Antikörper Napsin A war seinerzeit bei uns noch nicht etabliert.

Bei der Interpretation der Ergebnisse galt es, zwischen einem primären Lungenkarzinom und einer Lungenmetastase zu unterscheiden. Rein morphologisch war dieses bei TTF-1-Negativität der Tumorzellen nicht sicher möglich, wobei diese aber kein primäres Lungenkarzinom ausschließt. Insbesondere wurde gewürdigt, dass bei wiederholten bildgebenden und endoskopischbioptischen Untersuchungen während des 1-jährigen Verlaufes lediglich die Lungenraumforderung, die nunmehr eine Atelektase nach sich gezogen hatte, progredient gewesen war und sich ansonsten thorakal und abdominal keine Tumorhinweise ergeben hatten. In einem auswärtigen Vorgutachten war vier Monate vor unserer Begutachtung der BG bereits empfohlen worden, die vorhandenen Pleuraplaques als Brückenbefunde zur Anerkennung des Lungenkarzinoms als Berufskrankheit nach Nr. 4104 BKV zu werten, sofern sich internistisch oder radiologisch ein anderweitiger Primärtumor ausschließen ließe und sich in der Lunge ein radiologisch hinreichendes Tumorkorrelat für ein primäres Lungenkarzinom fände. Dieses war nunmehr aufgrund der Atelektasenbildung der Fall. Entsprechend haben wir in unserer Begutachtung die Voraussetzung für eine Anerkennung als Berufskrankheit als erfüllt angesehen.

\section{Diskussion}

$\nabla$

Der Respekt vor dem oft hohen Alter der Patienten und die schlechte Prognose von malignen Mesotheliomen und Lungenkarzinomen gebieten es gleichermaßen, bei einem tumorverdächtigen Lungen- oder Pleuraprozess bei einem Asbestexponierten zügig eine definitive Diagnostik herbeizuführen und sei- tens der BG gestellte gutachterliche Fragen nach einer Berufserkrankung schnell und möglichst noch zu Lebzeiten der Betroffenen zu klären. Da Pleuraergüsse ein sehr wichtiges und häufiges Leitsymptom beider Tumorerkrankungen sind und zur Entlastung der Dyspnoe oft ohnehin abpunktiert werden müssen, macht es grundsätzlich Sinn, ihre zytologische Untersuchung unter Einschluss adjuvanter Methoden der Pathologie als Grundlage der morphologischen Diagnostik zu verstehen. Gelingt auf diese Weise eine definitive Diagnosestellung, so ist die bei den häufig multimorbiden Patienten risikoreichere Durchführung einer Mediastinoskopie oder Thorakoskopie zur Gewinnung histologischer Proben aus unserer Sicht selbst für eine BG-Begutachtung nicht mehr notwendig. Vielmehr zeigt unsere Zusammenstellung von Fällen, dass es sich durchaus lohnt, vor der Einleitung invasiver Methoden zur Histologiegewinnung das bereits vorher vorliegende zytologische Material zu nutzen. Unsere Diagnostik gliedert sich dabei in folgende Schritte:

- Konventionelle Zytodiagnostik mit Stellung einer möglichst präzisen Primärdiagnose.

- Bei Tumorverdacht immunzytochemische Unterscheidung (insbesondere durch berEP4 und Calretinin) zwischen einem epithelialen Ursprung und einem mesothelialen Ursprung.

- Liegen epitheliale Zellen vor, so entspricht der Befund einer Pleurakarzinose, die immunzytochemisch weiter einzugrenzen ist, bei der Fragestellung eines Adenokarzinoms der Lunge insbesondere durch TTF-1- und Napsin A-Immunzytochemie.

- Ist der Prozess mesothelialer Natur, so ist die Unterscheidung zwischen reaktiven Veränderungen und einem malignen Mesotheliom durch die dargestellten adjuvanten Untersuchungen auf DNA-Aneuploidie, auf eine atypische AgNORVerteilung oder ggf. auch auf eine 9p21-Deletion durch FisH in der Regel möglich.

Dieses diagnostische Vorgehen hat sich in den vergangenen Jahren als sinnvoll herausgestellt, bedarf aber der Berücksichtigung von Randbedingungen.

Kürzlich in einem aktuellen Review [19] detailliert zusammengefasste Erfahrungen aus der Literatur zeigen beispielsweise, dass zwar die allermeisten Karzinome den Immunphänotyp [berEP4+/Calretinin -] und Mesotheliome in den meisten Fällen den Immunphänotyp [berEP4-/Calretinin+] aufweisen, wobei üblicherweise der jeweils positive Marker gleichmäßig und stark exprimiert wird. Nur selten kommen Fälle vor, in denen eine über Einzelzellen hinausgehende Ko-Expression beider Marker vorliegt, was dann die Interpretation der Immunreaktionen erschwert. Hier ist dann sinnvoll, mit EMA, WT1 oder Thrombomodulin andere Mesothelzellmarker oder mit HEA125 bzw. MOC-31 andere epitheliale Marker zur Differenzierung hinzuzunehmen, so wie wir es auch bei unseren Fällen bei begründeten Zweifeln gemacht haben.

Gleichermaßen markieren TTF-1 und Napsin A nicht alle Fälle von Adenokarzinomen der Lunge, sondern jeweils nur ca. 75\%85\% [20-24], sodass sich im Zweifelsfall ein Doppelansatz mit beiden Markern empfiehlt. Außerdem mehren sich in den letzten beiden Jahren auch Publikationen, in denen über die bekannten Expressionen von TTF-1 bei Schilddrüsenkarzinomen und von Napsin A bei Nierenzellkarzinomen hinaus auch bei anderen Karzinomen positive Fälle beschrieben wurden, z. B. nicht selten bei Adenokarzinomen des unteren Ösophagus [25] sowie selten bei Magenkarzinomen, Mammakarzinomen, Urothelkarzinomen, Prostatakarzinomen oder kolorektalen Karzinomen [26-29]. Auch wenn solche Pitfalls stets bedacht werden müssen, ist dennoch insbesondere ein TTF-1-Nachweis als sehr starker Indikator 
für ein Lungenkarzinom anerkannt und seine Anwendung explizit empfohlen $[19,26,30]$. Sowohl TTF-1 als auch Napsin A werden übrigens in malignen Mesotheliomen nicht exprimiert [17, $23-24]$.

Die meisten Versuche, zwischen reaktiven mesothelialen Veränderungen und malignen Mesotheliomen immunzytochemisch zu differenzieren, z.B. mit p53, bcl-2, EMA oder p170, wurden hingegen in einer Metaanalyse als nicht ausreichend verlässlich angesehen, da sich keine ausreichenden Daten für Sensitivität und Spezifität ergaben [31]. Ähnliches gilt auch für den Proliferationsmarker Ki-67 [32]. Eine gewisse Ausnahme wiederum stellt Desmin dar, mit dem sich entsprechende Werte von ca. 85\% - 90\% erzielen ließen [33-36]; ein Nachteil des Desmin ist allerdings, dass nicht etwa seine Expression, sondern sein Expressionsverlust auf ein malignes Mesotheliom deutet, was aus methodischen Gründen, insbesondere bei technischem Versagen der Färbung, zu Fehlinterpretationen führen kann.

Der Nachweis von DNA-Aneuploidie gelingt zwar lange nicht in allen Fällen von malignen Mesotheliomen, verfügt aber in der Abgrenzung gegenüber reaktiven mesothelialen Prozessen über eine Spezifität von 100\% [11,12,37-40]. Entsprechend lässt sich eine gewisse Zahl von Mesotheliomen bereits durch die Kombination Calretinin-Positivität/DNA-Aneuploidie eindeutig sichern, sodass dann im Grunde keine weiteren Untersuchungen mehr benötigt werden.

Bei weiter bestehendem morphologischen oder klinischen Mesotheliomverdacht schließt sich die AgNOR-Analyse an. Die Beurteilung der AgNOR-Zahl und AgNOR-Verteilung wurde bereits verschiedentlich an histologischen Schnitten von mesothelialen Läsionen durchgeführt, wobei allerdings verschiedene Parameter zur Anwendung kamen [41 - 45]; die histologischen Arbeiten, in denen größere Zahlen von Mesotheliomen und reaktiven mesothelialen Läsionen verglichen wurden, zeigten eine Sensitivität der AgNOR-Analyse von etwa $65 \%$ bei einer Spezifität von fast $100 \%$. Die bei uns gewählten Parameter der AgNOR-Satelliten und der AgNOR-Cluster gehen auf die Arbeitsgruppe um Crocker, der diese Methode als erster systematisch diagnostisch angewandt hat [15], zurück. Sie konnten an zwei Patientenkollektiven bei entsprechender Grenzwertziehung mit einer Sensitivität von $89,7 \%$ bzw. $95 \%$ und einer Spezifität von $97,1 \%$ bzw. $100 \%$ an zytologischem Material validiert werden [11,12], sodass diese Methode als sehr sinnvoller adjuvanter Ansatz in der Mesotheliomdiagnostik gelten kann.

Die technisch schwierigere und aufwendigere Fluoreszenz-in-situ-Hybridisierung zur Differenzierung zwischen reaktiven mesothelialen Veränderungen und einem Mesotheliom führen wir in der Routinediagnostik nicht regelhaft durch. Dem Nachweis einer Deletion an 9p21 kommt mit einer Sensitivität von 59\% bis $83 \%$ und einer Spezifität von 100\% zwar eine hohe Bedeutung zu [12,46 - 48], insbesondere da sich in einer vergleichenden Untersuchung mit Sonden für die Regionen 1p36, 14q32, 22q12, 5p15, 7p12 und 8q24 deutlich seltener FisH-detektierbare Veränderungen darstellen ließen [47]. Aufgrund des hohen technischen Aufwandes, der vergleichsweise hohen Kosten und der auch an den hier dargestellten drei Fällen doch eingeschränkten Sensitivität ordnen wir den Stellenwert der FisH-Untersuchung aber geringer als den von DNA-Zytometrie und AgNOR-Analyse ein.

Während die hohe Bedeutung der Zytologie für die Diagnostik insbesondere der nicht-kleinzelligen, aber auch der kleinzelligen Karzinome in der aktuellen S3-Leitlinie für Lungenkarzinome von 2010 [49] anerkannt ist, wird in der S2-Leitlinie zur Diagnos- tik und Begutachtung asbestbedingter Berufskrankheiten von 2011 [17] die Rolle der Zytologie bei der Diagnostik von malignen Mesotheliomen bislang noch als „nicht abschließend geklärt“ betrachtet. In der Tat kann die Entscheidung schwierig sein und bedarf dann, wie oben dargestellt, des Einsatzes adjuvanter Untersuchungsmethoden. Insgesamt belegen unsere hier dargestellten sieben rein zytologischen Fälle aus BG-Begutachtungen aber, dass unter Hinzuziehung von adjuvanten Methoden meistens eine so klare Diagnostik möglich ist, dass sich auch die sensiblen Fragestellungen der Berufsgenossenschaften entscheidungsreif lösen lassen und bei diesen oft alten und multimorbiden Patienten bei aussagekräftigem Pleuraerguss dann in der Tat auf eine histologische Diagnosesicherung ganz verzichtet werden kann. Entsprechend halten wir das „historische Misstrauen“ gegenüber der Zytologie, welches aus der Zeit vor der Etablierung solcher Anwendungen herrührt und die breite Überlappung morphologischer Zellveränderungen zwischen den reaktiven und den neoplastischen mesothelialen Läsionen reflektiert, für nicht mehr berechtigt. Ähnlich wird dieses auch in einem aktuellen internationalen Konsensus-Vorschlag zur Mesotheliomdiagnostik gesehen [9], der die Rolle der rein konventionellen Zytologie nach wie vor skeptisch, im Kontext moderner adjuvanter Anwendungen aber deutlich optimistischer und als mit der Histologie letztendlich gleichwertig beurteilt.

Dass einer unserer Fälle nicht ausreichend sicher diagnostiziert werden konnte (Fall 4), ist dabei nicht unerwartet - ganz natürlicherweise bleiben sowohl in der Zytologie, als auch in der bei der Beurteilung mesothelialer Läsionen nach wie vor ebenfalls diffizilen Histologie manchmal Fälle auch nach Anwendung des zur Verfügung stehenden breiten Methodenspektrums diagnostisch offen $[9,10,50]$. Wichtig ist dann, insbesondere wenn es um weitreichende gutachterliche Beurteilungen geht, bei denen nicht selten über ein erhebliches finanzielles Volumen entschieden werden muss, die entsprechenden differenzialdiagnostischen Schwierigkeiten klar zu benennen und im Gesamtzusammenhang aller zur Verfügung stehenden anamnestischen und klinisch-bildgebenden Informationen abgewogen zu bewerten.

\section{Interessenkonflikt}

$\nabla$

Die Autoren geben an, dass kein Interessenkonflikt besteht.

\section{Literatur}

1 Wagner JC, Sleggs CA, Marchand P. Diffuse pleural mesothelioma and asbestos exposure in the north western Cape province. Brit J Industr Med 1960; 17: 260-271

2 Schlegel H, Kempf E. Mesotheliome nach beruflichem Asbestkontakt. Soz Präventivmed 1982; 27: 220-222

3 Neumann V, Günther S, Müller K et al. Malignant mesothelioma - German mesothelioma register 1987-1999. Int Arch Occup Environ Health 2001; 74: $383-395$

4 Kamp DW. Subspeciality in translational medicine: Asbestos-induced lung diseases: an update. Transl Res 2009; 153: 143-152

5 Pesch B, Taeger D, Johnen $G$ et al. Cancer mortality in a surveillance cohort of German males formerly exposed to asbestos. Int J Hyg Environ Health 2010; 213: $44-51$

6 Robert Koch-Institut und die Gesellschaft der epidemiologischen Krebsregister in Deutschland e.V. (Hrsg). Krebs in Deutschland 2005/ 2006. Häufigkeiten und Trends. 7. Ausgabe. Berlin: 2010

7 Bundesregierung. Berufskrankheiten-Verordnung vom 31.10.1997 (BGBl I 1997: S. 2623), zuletzt geändert durch Verordnung vom 11.6.2009 (BGBl I; 2009: 1273-1276)

8 Rao RN. Mesothelioma. In: Shidham VB, Atkinson BF (Hrsg.) Cytopathologic diagnosis of serous fluids. Philadelphia: Saunders Elsevier; 2007: $107-113$ 
9 Husain AN, Colby T, Ordóñez $N$ et al. Guidelines for pathologic diagnosis of malignant mesothelioma: 2012 update of the consensus statement from the International Mesothelioma Interest Group. Arch Pathol Lab Med 2013; 137: 647-667

10 Henderson DW, Reid G, Kao SC et al. Challenges and controversies in the diagnosis of mesothelioma: Part 1. Cytology-only diagnosis, biopsies, immunohistochemistry, discrimination between mesothelioma and reactive mesothelial hyperplasia, and biomarkers. J Clin Pathol 2013; 66: $847-853$

11 Pomjanski N, Motherby H, Buckstegge B et al. Early diagnosis of mesothelioma in serous effusions using AgNOR analysis. Anal Quant Cytol Histol 2001; 23: $151-160$

12 Onofre FB, Onofre ASC, Pomjanski N et al. 9p21 deletion in the diagnosis of malignant mesothelioma in serous effusions additional to immunocytochemistry, DNA-ICM, and AgNOR analysis. Cancer 2008; 114: 204-215

13 Haroske G, Giroud F, Reith A et al. 1997 ESACP consensus report on diagnostic image cytometry. Part I: Basic considerations and recommendations for preparation, measurement and interpretation. Anal Cellul Pathol 1998; 17: 189-200

14 Öfner D, Aubele M, Biesterfeld S et al. Guidelines of AgNOR quantification - first update. Virch Arch 1995; 427: 341

15 Crocker J, Boldy DAR, Egan MJ. How should we count AgNORs? Proposals for a standardized approach. J Pathol 1989; 158: 185-188

16 Illei PB, Rusch VW, Zakowski MF et al. Homozygous deletion of CDKN2A and codeletion of the methylthioadenosine phosphorylase gene in the majority of pleural mesotheliomas. Clin Cancer Res 2003; 9: 2108 2113

17 Baur X, Clasen M, Fisseler-Eckhoff A et al. Diagnostik und Begutachtung asbestbedingter Berufskrankheiten. Interdisziplinäre S2-Leitlinie der Deutschen Gesellschaft für Pneumologie und Beatmungsmedizin und der Deutschen Gesellschaft für Arbeitsmedizin und Umweltmedizin. Pneumologie 2011; 65: e1 - e47

18 Welker L, Müller M, Holz O et al. Cytologic diagnosis of malignant mesothelioma - improvement by additional analysis of hyaluronic acid in pleural effusions. Virch Arch 2007; 450: 455-461

19 Ordóñez NG. Application of immunohistochemistry in the diagnosis of epithelioid mesothelioma: a review and update. Hum Pathol 2013; 44: $1-19$

20 Yatabe Y, Mitsudomi T, Takahashi T. TTF-1 expression in pulmonary adenocarcinomas. Amer J Surg Pathol 2002; 26: 767-773

21 Lau SK, Luthringer DJ, Eisen RN. Thyroid transcription factor 1: A review. Appl Immunohisto Mol Morphol 2002; 10: 97-102

22 Turner BM, Cagle PT, Sainz IM et al. Napsin A, a new marker for lung adenocarcinoma, is complementary and more sensitive and specific than thyroid transcription factor 1 in the differential diagnosis of primary pulmonary carcinoma - evaluation of 1674 cases by tissue microarray. Arch Pathol Lab Med 2012; 136: 163 -171

23 Bishop JA, Sharma R, Illei PB. Napsin A and thyroid transcription factor-1 expression in carcinomas of the lung, breast, pancreas, colon, kidney, thyroid, and malignant mesothelioma Human Pathology 2010; 41: $20-25$

24 Gomuluch BM. Die Wertigkeit von Napsin A in der Diagnostik primärer pulmonaler Adenokarzinome. Med. Diss. Ruhr-Universität Bochum; 2003

25 Aulakh KS, Chisholm CS, Smith DA et al. TTF-1 and Napsin A do not differentiate metastatic lung adenocarcinomas from primary esophageal adenocarcinomas. Proposal of a novel staining panel. Arch Pathol Lab Med 2013; 137: 1094-1098

26 Ordóñez NG. Value of thyroid transcription factor- 1 immunostaining in tumor diagnosis: A review and update. Appl Immunohistochem Mol Morphol 2012; 20: 429-444

27 Robens J, Goldstein L, Gown AM et al. Thyroid transcription factor-1 expression in breast carcinomas. Am J Surg Pathol 2010; 34: 1881 - 1885

28 Fernández-Aceñero MJ, Córdova $S$ et al. Aberrant TTF-1 expression in papillary high-grade urothelial neoplasm: case report and literature review. Rom J Morphol Embryol 2011; 52: 171 - 174

$29 \mathrm{Xu} B$, Thong N, Tan D et al. Expression of thyroid transcription factor-1 in colorectal carcinoma. Appl Immunohistochem Mol Morphol 2010; 18: $244-249$

30 Kawaguchi KR, Lu FI, Kaplan R et al. In search of the ideal immunopanel to distinguish metastatic mammary carcinoma from primary lung car- cinoma: A tissue microarray study of 207 cases. Appl Immunohistochem Mol Morphol 2013 [Epub ahead of print]

31 King J, Thatcher N, Pickering C et al. Sensitivity and specificity of immunohistochemical antibodies used to distinguish between benign and malignant pleural disease: a systematic review of published reports. Histopathology 2006; 49: $561-568$

32 Schönherr A, Bayer M, Böcking A. Diagnostic and prognostic value of Ki67 proliferation fraction in serous effusions. Cell Oncol 2004; 26: $57-62$

33 Hyun TS, Barnes M, Tabatabai ZL. The diagnostic utility of D2-40, calretinin, CK5/6, desmin and MOC-31 in the differentiation of mesothelioma from adenocarcinoma in pleural effusion cytology. Acta Cytol 2012; 56: 527-532

34 Hürlimann J. Desmin and neural marker expression in mesothelial cells and mesotheliomas. Hum Pathol 1994; 25: 753 - 757

35 Attanoos RL, Griffin A, Gibbs AR. The use of immunohistochemistry in distinguishing reactive from neoplastic mesothelium. A novel use for desmin and comparative evaluation with epithelial membrane antigen, p53, platelet-derived growth factor-receptor, P-glycoprotein and BCL-2. Histopathology 2003; 43: 231-238

36 Hasteh F, Lin GY, Weidner $N$ et al. The use of immunohistochemistry to distinguish reactive mesothelial cells from malignant mesothelioma in cytologic effusions. Cancer (Cancer Cytopathol) 2010; 118: 90-96

37 Friedman MT, Gentile P, Tarectecan A et al. Malignant mesothelioma. Immunohistochemistry and DNA ploidy analysis as methods to differentiate mesothelioma from benign reactive mesothelial cell proliferation and adenocarcinoma in pleural peritoneal effusions. Arch Pathol Lab Med 1996; 120: 959-966

38 Osterheld MC, Liette C, Anca M. Image cytometry: An aid for cytological diagnosis of pleural effusions. Diagn Cytopathol 2005; 32: 173-176

39 Motherby H, Marcy T, Hecker $M$ et al. Static DNA cytometry as a diagnostic aid in effusion cytology: I. DNA aneuploidy for identification and differentiation of primary and secondary tumors of the serous membranes. Anal Quant Cytol Histol 1998; 20: 153-161

40 Kayser K, Blum S, Beyer $M$ et al. Routine DNA cytometry of benign and malignant pleural effusions by means of the remote quantification server Euroquant: a prospective study. J Clin Pathol 2000; 53: 760 - 764

41 Wolanski KD, Whitaker D, Shilkin KB et al. The use of epithelial membrane antigen and silver-stained nucleolar organizer regions testing in the differential diagnosis of mesothelioma from benign reactive mesothelioses. Cancer 1998; 82: 583-590

42 Bethwaite PB, Delahunt B, Holloway LJ et al. Comparison of silver-staining nucleolar organizer region (AgNOR) counts and proliferating cell nuclear antigen (PCNA) expression in reactive mesothelial hyperplasia and malignant mesothelioma. Pathology 1995; 27: 1-4

43 Ayres JG, Crocker JG, Skilbeck NQ. Differentiation of malignant from normal and reactive mesothelial cells by the argyrophil technique for nucleolar organiser region associated proteins. Thorax 1988; 43: 366 370

44 Soosay G, Griffiths M, Papadaki L et al. The differential diagnosis of epithelial-type mesothelioma from adenocarcinoma and reactive mesothelial proliferation. J Pathol 1991; 163: 299-305

45 Vacharadze K, Burkadze G, Turashvili G et al. Argyrophilic nucleolar organizer regions in benign and malignant mesothelial lesions. Georgian Med News 2005; 128: 91 - 93

46 Chiosea S, Krasinskas A, Cagle PT et al. Diagnostic importance of 9p21 homozygous deletion in malignant mesotheliomas. Mod Pathol 2008; 21: $242-247$

47 Takeda M, Kasai T, Enomoto $Y$ et al. Genomic gains and losses in malignant mesothelioma demonstrated by FISH analysis of paraffin-embedded tissues. J Clin Pathol 2012; 65: 77-82

48 Monaco SE, Shuai Y, Bansal M et al. The diagnostic utility of p16 FISH and Glut-1 immunohistochemical analysis in mesothelial proliferations. Am J Clin Pathol 2011; 135: 619-627

49 Goeckenjan G, Sitter H, Thomas M et al. Prävention, Diagnostik, Therapie und Nachsorge des Lungenkarzinoms. Interdisziplinäre S3-Leitlinie der Deutschen Gesellschaft für Pneumologie und Beatmungsmedizin und der Deutschen Krebsgesellschaft. Pneumologie 2010; 64 (Suppl. 02): e1 - e164

50 Churg A, Galateau-Salle F. The separation of benign and malignant mesothelial proliferations. Arch Pathol Lab Med 2012; 136: 1217-1226 